\title{
Neuer therapeutischer Ansatz
}

Da das Mikrobiom mit vielen, teils chronischen Erkrankungen assoziiert ist, wird versucht, auch mit mikrobiotischen Präparaten therapeutische Effekte zu erzielen.

Ein neuer Ansatz bei der Therapie der Colitis ulcerosa (CU) ist die Stuhltransplantation, die in einer doppelblinden plazebokontrollierten Studie erste Erfolge gezeigt hat, erklärte Prof. Max Reinshagen, Klinikum Braunschweig. In der Studie hat der fäkale Mikrobiomtransfer von verschiedenen gesunden Spendern auf einen Patienten nach acht Wochen zur klinischen Remission der Erkrankung geführt [Paramsothy $S$ et al. Lancet 2017; 389: 1218 -28]. Auch eine Therapie mit speziellen Probiotika hat bei CU einen messbaren und mit 5-Aminosalicylsäure (5-ASA) vergleichbaren Effekt [Derwa Y et al. Aliment Pharmacol Ther 2017; 46: 389 - 400]. Die Kombination eines Präparats mit acht definierten, hoch- dosierten Bakerienstämmen (Innovall ${ }^{\oplus}$ CU) mit 5-ASA kann die Symptomatik merklich verbessern und die Zeit bis zur Remission im Vergleich zur Standardtherapie deutlich verkürzen.

Bei der Divertikelkrankheit hat die Gabe mikrobiotischer Präparate ebenfalls positive Wirkung gezeigt. Wie Prof. Joachim Labenz, Diakonie Klinikum JungStilling in Siegen, berichtete, kann sich die Divertikelkrankheit in leichten Beschwerden bis hin zu lebensbedrohlichen Komplikationen manifestieren. Unter der symptomatischen unkomplizierten Divertikelkrankheit (SUD) verstehe man persistierende oder rezidivierende Symptome einer Divertikulose, und keine Divertikulitis, wie Labenz mit Hinweis auf die Leitlinien betonte. Studien ergaben, dass bei SUD-Patienten ein Entzündungsmarker, die induzierbare Stickstoffmonoxid-Synthase (iNOS), signifikant überexprimiert wird [Turco F et al. United European Gastroenterol J 2017; 5 (5):715-25]. An Biopsien konnte gezeigt werden, dass die $\mathrm{Zu}$ gabe von Lactobacillus casei $\mathrm{DG}^{\circledast}{ }^{(\text {Innoval }}{ }^{\circledast}$ SUD) die iNOS-Expression signifikant auf den Basalwert senkt $(\mathrm{p}<0,05)$.

Beim Reizdarmsyndrom (RDS) zielen die verfügbaren Therapien auf die verschiedenen Leitsymptome wie Schmerzen, Obstipation, Meteorismus und Diarrhoe ab, erläuterte Prof. Heiner Krammer, niedergelassener Gastroenterologe aus Mannheim. Mikrobiotika können jedoch das Grundproblem der Dysbiose adressieren, wobei die Wahl des Stammes nach der Symptomatik erfolgen sollte. Nur Lactobacillus plantarum 299v (Innovall ${ }^{\circledR}$ RDS) hat sich wirksam bei allen RDS-Leitsymptomen, insbesondere beim Schmerz-/Bläh-Typ erwiesen [Ducrotte P et al. World J Gastroenterol 2012; 18: $4012-18]$.

Ursula Hilpert

Pressekonferenz "Modulation des Darmmikrobioms - von der Pathophsysiologie zu neuen Therapiekonzepten“; Viszeralmedizin 2017, 14.9.2017, Dresden; Veranstalter: Microbiotica

\section{Morbus Crohn}

\section{Bessere Ergebnisse mit Treat-to-Target}

Die Optimierung der Behandlungsergebnisse chronisch entzündlicher Darmerkrankungen (CED) sei erforderlich, um irreversible Organschäden zu verhindern sowie weniger Hospitalisation und Operationen, bessere Lebensqualität und weniger Therapienebenwirkungen zu erreichen, betonte Prof. Axel Dignass, Agaplesion Markus Krankenhaus, Frankfurt am Main. Gründe für das Ausbleiben des gewünschten Therapieerfolgs seien der variable Verlauf der CED, unzureichende Verlaufsprädiktoren, zu späte Diagnose/ Behandlungsbeginn sowie nicht wirksame oder nicht optimal eingesetzte Therapien. Für die Optimierung des Outcomes bei CED forderte Dignass den Einsatz optimaler Therapien zu einem frühen Zeitpunkt, die Nutzung validierter, objektiver Endpunkte zur Verlaufskontrolle, die laufende Anpassung der Therapien bis die Endpunkte erreicht sind (Treat to Target) und die Rundumbeurteilung des CED-Patienten, einschließlich seiner psychosozialen Bedürfnisse.
Da Morbus Crohn (MC) eine progressive Erkrankung sei, könne eine frühe Intervention das Fortschreiten verhindern und eine Krankheitsmodifizierung ermöglichen, betonte Dignass. Dazu müssten die optimalen Behandlungsfenster der Erkrankung erkannt und genutzt werden. Als Therapieziele nannte er die histologische Remission, die Mukosaheilung, die steroidfreie Remission, die klinische Remission und verbesserte Symptome.

Den positiven Effekt einer engmaschigen Kontrolle und des Treat-to-TargetKonzepts bei MC belegt die prospektive, offene, multizentrische und aktiv kontrollierte 48-wöchige Phase-III-Studie CALM, in der 244 erwachsene, immunsuppressiva- und biologikanaive Patienten mit mittelschwerem bis schwerem aktivem MC (mittlere Erkrankungsdauer ein Jahr) mit konventionellem Management (KM) und der Treat-to-TargetStrategie unter Adalimumab (Humira ${ }^{\circledR}$ verglichen wurde, berichtete Prof. Stefan Schreiber, Universität Kiel. Treat-to-Tar- get beinhaltete die engmaschige Kontrolle (Tight control) der Patienten mit Biomarkern wie C-reaktives Protein (CRP) und fäkalem Calprotectin (FC) zur Bestimmung der Entzündungsaktivität.

In der Treat-to-Target-Gruppe erreichten $45,9 \%$ der Patienten den primären Endpunkt Mukosaheilung (CDEIS $<4$, keine tiefen Ulzerationen zu Woche 48), in der KM-Gruppe lag der Anteil bei 30,3\% $(\mathrm{p}=0,01)$ [Colombel JF et al. Gastroenterology 2017; 152( Suppl 1): 155]. Darüber hinaus erreichten Patienten mittels Treatto-Target nach 48 Wochen signifikant häufiger eine steroidfreie Remission (ein sekundärer Endpunkt) als Patienten unter KM (59,8\% vs. $39,3 \%$; $p=0,001)$. CALM sei die erste Studie, die belegt, dass ein Treat-to-Target-Konzept zu besseren Ergebnissen als die konventionelle, symptombasierte Therapiesteuerung führt. Dabei habe die Nutzung von CRP als Treatto-Target-Ansatz keine erhöhten Sicherheitssignale ergeben resümierte Schreiber.

Dr. Thomas Riedel

Symposium „Satellitensymposium „Mission Zukunft - CED-Therapie im Jahr 2020", Viszeralmedizin 2017, 14. September 2017, Dresden; Veranstalter: AbbVie 